\title{
Acute Humoral Allograft Nephropathy
}

National Cancer Institute

\section{Source}

National Cancer Institute. Acute Humoral Allograft Nephropathy. NCI Thesaurus. Code C114841.

A sudden onset of B-cell mediated immune response occurring after transplantation, directed against donor kidney alloantigens. 